\title{
The expert evaluation systems in drug development
}

\author{
Elena Zhilyakova ${ }^{1, *}$, Ok Savin ${ }^{2}$, Alexandra Baskakova $^{1}$, and Natalia Avtina ${ }^{1}$ \\ ${ }^{1}$ Belgorod State National Research University, Belgorod, Russia \\ ${ }^{2}$ Royal University of Agriculture, Dangkao Section, southwest Phnom Penh, Cambodia
}

\begin{abstract}
This study investigates the unique properties and criteria of use intellectual systems in drug delivery. The traditional methods of pharmaceutical drug development are limited in the field of usability. In connection with the above mentioned, the task of development and creation of the intellectual system allowing to choose active pharmaceutical ingredients, auxiliary substances, which will be based on the expert system and information base of knowledge in the field of pharmaceutical production and it should be of current scientific and practical relevance. The most commonly used type of intelligent systems today are expert systems, which are computer systems capable of partially replacing a highly qualified specialist in his or her field of competence through the knowledge previously obtained from him or her. That is why expert systems are accepted to be considered together with knowledge bases, which are models of specialists' behavior in a certain field, and for decision-making procedures of logical conclusion are applied. The field of application of expert systems is absolutely unlimited. A vivid example is their use in diagnostic tasks of modern medicine.
\end{abstract}

\section{Introduction}

Pharmaceutical development (FD) is an essential stage in the life cycle of medicines. According to the modern provisions adopted in countries with developed pharmaceutical industry, FD is called upon to implement the principles of planned quality. Various aspects of pharmaceutical development are considered in documents adopted by international organizations.

Methodological guidelines for pharmaceutical development contain a document adopted by the International conference on harmonization of technical requirements for registration of medicines for humans ICH Q8, reflecting the stages, content, logic and requirements [1]. According to this document planned quality, or quality by development, provides "a systematic approach to development based on sound scientific evidence and product quality risk management that begins with the definition of objectives and focuses on understanding the product and process and controlling the latter". Only from the point of view of the planned quality FD will be a kind of guarantee for the production of quality, efficient and safe medicines [2].

Today, one of the least standardized and formalized stages of development of the finished dosage form is the stage of pharmaceutical development. The purpose of pharmaceutical development is to create a medicine of appropriate quality and justify the process of its production with the given characteristics. Principally, the adoption of the QbD approach should ensure the creation of a medicine of a given quality with minimal risk [3]. Central to the QbD approach are experimental studies, in which, in turn, the analytical and informational support of research necessary to control the technological process itself and the product produced are of paramount importance.

\section{Problem statement}

Currently, there is a need to classify and systematize knowledge in this area in order to create effective tools to reduce development time, which is a limiting stage when a new medicine enters the market. In connection with the above mentioned, the task of development and creation of the intellectual system allowing to choose active pharmaceutical ingredients, auxiliary substances, which will be based on the expert system and information base of knowledge in the field of pharmaceutical production and it should be of current scientific and practical relevance.

The most commonly used type of intelligent systems today are expert systems, which are computer systems capable of partially replacing a highly qualified specialist in his or her field of competence through the knowledge previously obtained from him or her [4]. That is why expert systems are accepted to be considered together with knowledge bases, which are models of specialists' behavior in a certain field, and for decisionmaking procedures of logical conclusion are applied. The field of application of expert systems is absolutely unlimited. A vivid example is their use in diagnostic tasks of modern medicine.

These systems implement procedures to identify the relationship between failures in the human body and the potential causes of their occurrence, which allows physicians to make more accurate diagnoses, and take into account a greater number of factors [5]. Besides, the main directions of application of expert systems are planning, the control and management of resources in 
areas of logistics, marketing and management of the enterprises that allows to optimize considerably processes of manufacture, distribution, etc., including by working out of medical products as the given processes occupy a significant enough part of life cycle of manufacture of medical products [6].

\section{Purpose of the study}

The key stage in the implementation of any expert system is a clear understanding and adherence to the principles of information support for process control systems.

According to the literature, there are several methods and models for assessing information systems, including expert systems based on databases. The disadvantages of the assessment methods described earlier are their lack of systematization and complexity of application, lack of uniform terminology and insignificant practical application.

\section{Results and discussion}

The results of expert systems should be evaluated according to their characteristics such as usability, ease of improvement and impact on users who do not use expert systems. However, most authors identify their efficiency and effectiveness as the most important indicators of expert systems. Efficiency is understood as a level of achievement of the purpose in the set conditions, connected with results of decision-making, received on an output, that is efficiency of correlation of inputs (resources) and outputs [7]. Efficiency means the use of the smallest amount of resources, however, on the other hand, efficiency can be verified by feedback from users of the expert system. This method of evaluating the efficiency of an expert system allows to make sure that it meets the interests of users and meets their needs [8].

Cascant and others also describe user satisfaction as an indicator of high efficiency of the expert system, which allows increasing the number of users using the expert system to solve their problems [9]. Evaluation can be presented as a process that allows increasing the use, quality and usefulness of the expert system.

An important point in the evaluation of expert systems is the necessity to consider the expert system not as an isolated object, but as a working tool used to achieve certain goals. The review of literature has revealed some parameters for the evaluation of expert systems. These parameters clearly show that the productivity of expert systems should be evaluated from the point of view of influence of users, system and organization, where the expert system is implemented, on each other. P. Miranda and co-authors in their research note that in this case users are responsible for the performance of the tasks [10].

Mauldin, E. and the co-authors emphasize in their research the fact that the interdependence between these objects acts as a determining factor for the realization of a given task [11]. Expert systems help to reduce the time spent on medicine development as well as the burden on researchers.

Expert systems generate profits for the organizations in which they are implemented, for example, by leading to more accurate and informed decision-making and by reducing time spent on tasks [12]. Users, with the proper use of expert systems, increase productivity in the organization.

As it was described earlier, the majority of methods of an estimation of expert systems on the literary data have no structured parameters of an estimation of expert systems. Parameters which can be used at an estimation of productivity of expert systems, have been grouped for their further identification and systematization according to a role of the subjects participating at an estimation of expert system: the user, expert system and the organization [13].

Table 1 presents the parameters and criteria for evaluation of expert systems. From the point of view of the user of the expert system, three parameters can be distinguished, according to which the system can be evaluated: usability, utility, quality. The parameter "usability" is described by foreign authors as ease of use of the system [14].

One more parameter, considered from the user's point of view, for estimation of productivity of expert system is "utility". The "utility" is understood as what benefit the user of the expert system gets. Performance of the system should be estimated according to its usefulness. In order to determine whether the expert system meets expectations, it is necessary to understand whether the expert system will be used to solve the user's tasks despite the existing difficulties or inconveniences.

The technical definition established by the International organization for standardization (ISO) describes the term "quality" as usability. This suitability needs to be confirmed $[15,16]$. Table 2 presents the criteria that are related to the criterion of "quality": reliability, quality of the obtained solution of the problem, consistency, update time, coherence, response time.

From the system point of view, two parameters have been defined for the evaluation of expert systems: interface and structure. The interface and structure parameters allow the criteria to be identified according to the software and hardware aspects.

The "interface" parameter corresponds to the system design and should provide access to information. The interface in the expert system is a set of characteristics that users apply when interacting with the system. Thus, the interface is all that is available to the user to control the system how the system should react to the user's actions. To evaluate the "interface" parameter five criteria are defined: ease of use, quality of proposed solutions, appearance, ease of search, quality of data input and display.

The next parameter related to the system is structure. Criteria for evaluation of this parameter are stability, number of errors, processing of operations, history, processing time of operations.

From the point of view of the organization that implements expert systems, there are two parameters that have been defined to evaluate expert systems: productivity and profit. Criteria for evaluation of the parameter "productivity": motivation, task optimization, profit, reports, efficiency, cost and efficiency. These criteria evaluate aspects related to financial turnover and economic activities, which are mentioned in the studies. 
The parameter "profit" emphasizes the connection between the expectations associated with investments in the development of expert systems and the expected income. The main criteria of this parameter are

Table 1. The parameters and criteria for evaluation of expert systems

\begin{tabular}{|c|c|c|c|}
\hline Subjects & Parameters & \multicolumn{2}{|c|}{ Criteria } \\
\hline \multirow{7}{*}{ User } & \multirow[t]{3}{*}{ Usability } & Study & Efficiency \\
\hline & & Allowable error & Satisfaction \\
\hline & & Performance & Expediency \\
\hline & Utility & Scale & Expediency \\
\hline & Quality & Credibility & $\begin{array}{l}\text { The quality of the } \\
\text { obtained solution to } \\
\text { the problem }\end{array}$ \\
\hline & & Coherence & Update time \\
\hline & & Coherence & Response time \\
\hline & Interface & Ease of use & $\begin{array}{c}\text { Quality of proposed } \\
\text { solutions }\end{array}$ \\
\hline & & Appearance & Search simplicity \\
\hline Expert system & & Quality of data input and display & \\
\hline & Structure & Stability & Number of mistakes \\
\hline & & Processing operations & History \\
\hline & & Operations Processing Time & \\
\hline & Productivity & Motivation & Task optimization \\
\hline & & Profit & Reports \\
\hline Ororanization & & Performance & Cost \\
\hline Organization & & Efficiency & \\
\hline & Profit & Competitiveness & Return on investment \\
\hline & & Cost reduction & \\
\hline
\end{tabular}

Table 2. The related criteria to the "quality" parameter

\begin{tabular}{|c|c|}
\hline Criteria & Description \\
\hline Credibility & User confidence in system solutions \\
\hline Coherence & The integrity of the presentation of information in the system \\
\hline Coherence & The ability of the system to correspond to reality (relevance of decisions) \\
\hline $\begin{array}{l}\text { The quality of the obtained solution to the } \\
\text { problem }\end{array}$ & Quality of problem solving \\
\hline Update time & $\begin{array}{l}\text { The ability of the system to make decisions in a short time, and also to be updated in the } \\
\text { decision-making process }\end{array}$ \\
\hline Response time & $\begin{array}{l}\text { The time during which the user expects a response from the system, from the time of the } \\
\text { request to the decision }\end{array}$ \\
\hline
\end{tabular}

\section{Conclusion}

Thus, the offered criteria of an estimation of expert systems can be used at their working out and introduction in a production cycle of the organizations which are engaged in working out of medical products that will allow to reach growth of the income and decrease in expenses for decision-making by ordinary employees that will increase their productivity and will reduce labor inputs. competitiveness, cost minimization and return on investment. 
3. J.A. Selekman, J. Qiu, K. Tran, J. Stevens, V. Rosso, E. Simmons, Y. Xiao, and J. Janey, Annual Review of Chemical and Biomolecular Engineering, 8, p. 525-547, (2017)

4. M. Kanatov, L. Atymtayeva, and B. Yagaliyeva, "Expert systems for Information Security Management and Audit. Implementation phase issues," (IEEE, C. SCIS\&ISIS. Kitakyushu, Japan, 2014)

5. K. Fielding, A. Long, N. McSweeney, M. Payne, and E. Smoraczewska, Drug Design Strategies: Quantitative Approaches, 11, 279-311 (2012)

6. M. Grechanik and B.M.M. Hossain, "Enhancing performance and reliability of rule management platforms ICPE 2015 - Proceedings of the 6th ACM/SPEC International Conference on Performance Engineering,” p. 187-198 (2015)

7. G. González Rodríguez, J.M. Gonzalez-Cava, and J.A. Méndez Pérez, Journal of Intelligent Manufacturing, 31 (5), 1257-1273 (2020)

8. C. Anumba and D. Scott, Structural Survey Journal, 19(5), 222-232. (2001)

9. P. Cascante, M. Plaisent, L. Maguiraga, P. Bernard, Information Resources Management Journal, 15(4), 64-78 (2002)
10. P. Miranda, P. Isaias, and M. Crisostomo, "Expert systems evaluation proposal," In: Smith, M.J., Salvendy, G. (eds.), (HCII, LNCS, Springer, Heidelberg, 2007)

11. E. Mauldin, Journal of Information Systems, 1, 1941, (2003)

12. G. Guida and G. Mauri, IEEE Transactions on Knowledge and Data Engineering, 5(2), 204-224 (1993)

13. A. Cojocariu, A. Munteanu, and O. Sofran, Computational Economics, (2005), 0510002, EconWPA

14. D. Waterman, A Guide to Expert System. Reading (Mass.) etc.: Addison-Wesley, Cop., 1986.ISO/IEC 25010:2011(en) Systems and software engineering - Systems and software Quality Requirements and Evaluation (SQuaRE) System and software quality model.

15. N. Bevan, J. Carter, J. Earthy, T. Geis, and S. Harker, "New ISO Standards for Usability, Usability Reports and Usability Measures," in International Conference on Human-Computer Interaction, Part I, M. Kurosu, ed., 9731, p. 268-278 (2016) 